
\title{
28 Research Square \\ Effects of eight-week progressive resistance training on physical fitness and psychological health in adolescent males
}

José Afonso

University of Porto

Masoud Sadeghi

Razi university

Omid razi

Razi university

Alexandre Martins

University of Poro

Abdolhossein Parnow ( $\sim$ parnowabdolhossein@gmail.com )

Razi university

\section{Research Article}

Keywords: resistance training, programming, linear training, undulating training, mental health

Posted Date: September 27th, 2021

DOl: https://doi.org/10.21203/rs.3.rs-940934/v1

License: (9) This work is licensed under a Creative Commons Attribution 4.0 International License. Read Full License 


\section{Abstract}

Introduction: Physical fitness and psychosocial health might improve through resistance training programs. However, there has been controversy regarding the efficacy of different exercise programs. To compare the effect of 8 weeks' resistance training with linear and undulating programming on physical fitness and mental health.

Methods: 29 healthy and untrained students (age: 16.06 \pm 0.83 ) were randomly divided into three groups: (1) control ( $n=6)$, (2) linear programmed resistance training (LPRT) ( $n=12)$, and (3) undulating programmed resistance training (UPRT) $(n=11)$. After two weeks of familiarization, 1 RM values were obtained. Thereafter, experimental groups attended a resistance training program of 3 sessions per week for 8 weeks. Both groups had a similar plan until the fourth week. Then, intensity was increased up to $70 \%$ of 1 RM on a weekly basis for the LPRT group, and varied on a daily basis for the UPRT group. Upperand lower-extremities strength and endurance, balance, speed, explosive-power, lean body mass, general mental health (GSQ) and physical self-concept (PSCQ) were measured.

Results: Experimental groups produced significant improvements in physical outcomes, in comparison with controls. There were no improvements in GSQ and PSCQ. There were no statistically significant differences between the two programs.

Conclusion: Both linear and undulating programs improved physical fitness of previously sedentary adolescent boys to a similar extent. For practical purposes, as long as loads are individually adjusted, the type of program may not be relevant in beginner practitioners. Furthermore, both models were ineffective in producing changes in the psychosocial variables.

\section{Introduction}

Physical fitness is an integrated measurement of the majority of body functions such as skeletal muscles, cardiovascular, blood circulation, psycho-neuronal and hormonal-metabolic activities [1]. When physical fitness is assessed, hence, the functional condition of all body systems is actually examined and that is a reason why the physical fitness is considered a relevant factor in chronic diseases [1]. Chronic diseases are usually progressed slowly and are resulted from genetic, environment or poor life-style factors [2]. Besides, increasing of various chronic diseases like respiratory disease, obesity, cancer, cardiovascular disease, diabetes and stroke. It should be mentioned that quality of life (QOL) is reduced in future generations [2]. By incorporating physical activity or exercise in one's life-style, physical fitness as a leading health index could be improved [1,2]. Additionally, it is worth noting that childhood and adolescence are very sensitive life periods, since lifestyle and exercise behaviors established in those periods may impact adult behaviors and health status $[1,3]$.

In the context of exercise interventions, several studies have shown that resistance training (RT) is an effective procedure to increase muscle mass and strength, improve performance and stimulate healthy life-style in adolescents [4,5]. Furthermore, despite discussions concerning potentially harmful effects of 
RT in youth [6-9], it has been generally recognized that it can improve psychosocial health such as depression, anxiety, fatigue, QOL, and self-estimation [10-13]. Even though there are compelling documents considering the effectiveness of resistance training in adolescents, the various factors are contributed to adaptive processes following RT sessions.

Moderator variables and RT model are two involving factors [14]. Moderator variables include training principles such as intensity, frequency, type, volume and periodization and so on [15]. Of those, RT programming has assumed a special importance and are usually more effective than constant (nonvaried) RT programs [14, 16-19], even if this trend is not universal [20,21]. Linear and undulating programming are two common methods of engaging in $\mathrm{RT}[18,22]$. Linear programming is usually associated with a program initiated with high volume and low intensity and gradually by program progression the volume decreased and to intensity increased and typically lasts several months, even if the term linear has been coined a posteriori and was not applied by original periodization models. In undulating programming, the volume and intensity can be changed in every single session [17], thus providing greater diversity and avoiding load monotony.

To the best our knowledge, there are no investigations comparing linear and undulating periodized RT, in the critical period of adolescence and evaluating both physical and psychological factors. Consequently, the current investigation aimed at examining the effect of 8-week linear and undulating periodized trainings on physical fitness and psychological factors in male adolescents.

\section{Methods}

\section{Experimental design and sample}

The current study was quasi-experimental pre- and post-test design. The participants were healthy but untrained high-school students with an age range of 14 to 18 years-old and after invitation, the details were informed to them. Thereafter, 29 out of 90 participants accepted to contribute to the study and the consent form was filled out by their parents. Furthermore, they were screened in sports history and health condition by self-reported questionnaire. The inclusion criteria were: (1) being aged between 14 to 18 years, (2) not having diseases such as asthma, diabetes or, thalassemia, (3) not having any movement disabilities, (4) not having any injuries, (5) not having any RT experience at least during the last 6 months, and (6) not consuming any dietary supplementations like multi-vitamins, creatine and protein before and during training. Moreover, the exclusion criteria were: (1) no regular attending to training sessions outside the scope of this study, (2) voluntary exit from the study, (3) consumption of dietary supplements during training course, and (4) disability in continuing the training protocol due to injury.

Before assessing the participants' one-maximum repetition (1RM) in leg press and chest press, all groups experienced a familiarization program for two weeks, with three sessions per week. Their 1RM was measured and recorded in the last session of familiarization period. Pre-test rates of considered variables were measured after 48 hours from recording the 1RM. Participants were randomly divided into three 
groups: (1) linear programmed RT (LRT) ( $n=12)$, (2) undulating programmed RT (UPRT) $(n=11)$, and (3) control $(n=6)$. The experimental groups implemented an 8-week training program that was specific for each group. Upon reaching to the final of the 4th week (the middle-point of the 8-week training program), $1 \mathrm{RM}$ was measured for every single group once again and the remaining four-week program was set with respect to the new $1 \mathrm{RM}$. Collectively, the post-test rates of consider variables were measured as equal conditions as pre-test measurement 48 hours after the last training session of 8-week training program. It is worth noting that the current study was approved by ethics committee of Razi University in accordance with the declaration of Helsinki (updated version of 2013).

\section{Assessments of physical fitness}

Before conducting all tests, a 5-10 min period was devoted to warm-up phase. Muscle strength was obtained by performing the 1RM test in both pre- and post-tests. However, in order to obtain the 1RM from more repetitions, the $1 \mathrm{RM}$ indirectly identified via set the repetitions numbers and work-load which is shifted based on Brzycki Formula [23]: 1RM = weight lifted/(1.028 - no. repetitions*0.0278).

To measure the strength of lower and upper extremities, the leg press and chest press were utilized, respectively. A two-week familiarization period was used before the testing. For chest press, participants used middle grip-width. The chest press ended with the greatest weight or the number of repetitions that participants could perform to the mid-chest and back to the starting position. In the leg press, individuals were instructed to sit on the machine with their heels flat and spaced at hip width. Both in the bench press and leg press, the execution of the greatest weight or the number of repetitions performed by each participant during the test were accounted for calculating the strength of lower and upper extremities [24].

The muscular endurance of shoulder girdle, furthermore, was assayed by push-up test and the correct number of actions was counted and then recorded for participants. Additionally, wall-squat was utilized to measuring the muscular endurance of lower extremities. In this case, asked from the participants that get close to the wall and leaning their back to it with feet shoulder width and 2 feet from the wall. Then, the participant must slide his back down the wall until reaching the proper position, i.e., at 90-degree angle to both the back and lower legs. As long as the participant could able to hold this position the time for that person is recorded [25].

Y balance test (YBT) was used to measure the dynamic balance. The leg length (right side) was measured prior performing the main test, since the knowing this measurement is needed to results calculations. Before starting the measurement procedure, the $Y$ like-line was drawn by using white strap with a rectangular stance in center. For measuring the dynamic balance, the participants must place on central white stance with standing on one leg. Then, subjects by extending his predominant foot in anterior direction attempt to push his leg as far as he can while maintaining balance. Such procedure for other directions (posterior-medial and posterior-lateral) must be performed. The free foot should be returned to the starting position under control. The whole performed protocol in one foot must be conducted for another foot. This test was conducted 3 times for each foot and then the dynamic balance 
for each foot was separately calculated as follow [26]: scores summation of three directions/foot length ${ }^{3}$ $\times 100$.

The jump Sargent test was used to measure explosive-power. Before the assessment, participants were given the necessity to take part to warm-up program consist of 10 squats, 10 alternating high knees and 2 min running in place. Following verbal explanation and physical demonstration by an exercise physiologist, from participants requested to place close to the calibrated wall with stretching up their hands and the place of their fingertips was marked before jumping. Then, requested from them to squat down and jump up and the highest point on the wall after jumping was again marked. The distance between two positions (stance and jumping marked points) was measured in centimeter and finally explosive-power of feet was obtained in Watts as following [27]: Power $=[\sqrt{ }(4 / 9)]$ * weight $(\mathrm{kg}) * \sqrt{ } \mathrm{VJ}(\mathrm{m})$ $\star 9.81$.

The speed was measured by 30-meter speed test. A 30-meter distance was identified by a calibrated strap and the start and finish points was marked. Upon starting the participant must run as quickly as he can. Two trials with 5 min recovery were recorded for each individual and the average time took accounted as their records [25].

Regarding free fat body mass index (FFMI), the height and weight were obtained and body fat percent was also evaluated from right side of 2-point skinfold of triceps and calf by utilizing a caliper (Seyhan, South Korea). Assessments were made a highly experienced technician in collecting skinfolds. Body fat percent and FFMI were calculated as follows for black or white boys 6 to 17 years-old (with predictive accuracy of $\pm 8.6 \% \mathrm{BF})[28,29]$ : (i) $\% \mathrm{BF}=0.735 *\left(\sum 2 \mathrm{SKF}\right)+1.0 ; \sum 2 \mathrm{SKF}$ (triceps + calf); (ii)

FFMI $=\frac{\text { mass }(\mathrm{kg}) \times((100-\text { fat } \%) / 100)+6.1 \times(1.8-\text { height }(\mathrm{m}))}{\text { height }^{2}}$

\section{Assessments of psychological health factors}

General health question questionnaire

The general health questionnaire-28 (GHQ-28) questionnaire was utilized for evaluating the four components of physical symptoms, anxiety signs, disturbing in socio-function and depression. The questionnaire is based on a Likert scale and the scoring ranges from $0-3$, with higher values indicating worse conditions. The lower- and upper-score limitations of the questionnaire are 0 and 84 . The questionnaire's reliability using alpha-Cronbach test was 0.74 . In the current study, the participants were initially explained how to fill-out the questionnaire, which was filled in two occasions: before engaging with the main protocol and after finishing the 8-week intervention. 
In according to Cronbach formula, the reliability coefficient for this questionnaire was 81.0. The utilization of current four-factor questionnaire is usually to evaluate the assuming of ones about his/her-self body, health, physical appearance and body-strength. Physical self-concept questionnaire (PSCQ) comprises 8 questions, each containing 5 options. The higher the score, the better the self-concept.

\section{Training protocols}

\section{Linear programmed resistance training (LPRT)}

This training included 8-week training and 3 sessions /week. 2 weeks was devoted to participants' familiarization to the program. After defining 1RM for every given exercise at the last session of familiarizing period, the pre-test of consider variables was measured. Each training session lasted $60 \mathrm{~min}$ and included warm-up followed by chest-press, barbell squat, Bulgarian squat, barbell curl, barbell triceps extension, Underhand Cable Pulldowns, plank, hyperextension (back extension) and finally cool-down. The training was started with $30 \%$ of $1 \mathrm{RM}$ and in fourth week was reached $50 \% 1 \mathrm{RM}$. At the end of week 4 , the 1RM was again evaluated for every single of the movements and the new obtained 1RM was based as the training's 1RM of the remaining of 4-week (week 4 to end of week 8 ). Thus, the training program was incremental from 60 to $70 \%$ of $1 \mathrm{RM}$ during the second half of program (Table A.1). Eventually, the post-test measurements were obtained after 48 hours of the last session of 8-week training protocol.

\section{Undulating programmed resistance training (UPRT)}

The whole procedure of this training such as duration, session frequency, the time and the training movements was similar to linear programmed resistance training. However, how to implement the volume and intensity of training was the only difference; such that, volume and intensity were variable per session from fourth week onwards. Thus, participants are involved in incremental training protocol each session with new intensity and volume over week for four remaining weeks (Table A.1). It should be mentioned that 1RM was also measured by finishing the fourth week. Additionally, the postmeasurements of the variables were obtained 48 hours after of the last session of 8-week training protocol.

*** Table A.1 $1 * * *$

\section{Data analysis}

The Shapiro-Wilk test was used to assess normality of data. Mixed ANOVA was also utilized to measuring the significant differences between and within groups and Post hoc test was conducted to assess paired comparisons. The significant level was set at 0.05 . All data analyzed by SPSS software, version 22. To identify the changing percent, the following formula was applied: $\%$ change $=$ (post value pre value) $* 100$.

\section{Results}

Some demographic properties of participants were shown in Table A.2. 
The mixed ANOVA results considering upper- and lower-extremities strength indicated that there was statistical significance for time, groups and time $\times$ group interaction, large effect size. By further Post hoc analyzing, it has been shown that there were no significant differences among training groups $(p>0.05)$, while such differences were observed with comparing to control group $(p<0.001)$ (Table A.3), large effect sizes. In light of statistical significance of time, the pre-and post-measures of two training groups were significantly different $(p<0.001)$, with large effect sizes in both factors.

However, there were statistical significances in time, group and interaction in terms of upper-extremities endurance, such significant differences were not observed in lower-extremities endurance, excepting in pre- and post-measures (Table A.3). Detailed analyzing of upper endurance by Post hoc was shown that there were significant differences in UPRT with control group $(p<0.05)$, large effect size, but such differences were not in LPRT group ( $p>0.05)$. Importantly, there was statistically significant difference between LPRT and UPRT ( $<$ 0.001) (Table A.3). In respect to time effect, it has been identified that preand post-measures of upper-extremities endurance in two training groups were significant difference following 8-week training protocol $(p<0.001)$, large effect sizes.

$\star \star \star *$ Table A.3 ***

The findings of balance, which is measured by Y-balance test in right and left legs, defined that there was significant time $\times$ group interaction and Post hoc test, then, revealed a significant measure between two training groups with those control group ( $p>0.001$, Table A.3). Accordingly, the differences were precisely present between LPRT and UPRT groups $(p<0.001)$, large effect sizes. It has also been found that the preand post-measures of right and left balance in all training groups were significantly changed after 8-week protocol $(p<0.001)$, large effect size.

As depicted in Table A.3, regarding to explosive-power the pre- and post-measures of UPRT group were significantly different consequence of given 8-week training protocol $(p=0.04)$, relatively large effect size.

There was a statistical significance in interaction in terms of speed factor $(p=0.02)$, large effect size. After analyzing the main time effect and group differences by repeated measure test and Post hoc test, respectively, it was identified that there was no significant difference between groups as well as the preand post-measures of each group had the same result $(p>0.05)$.

Regarding FFMI, there was statistical significance in time, group and interaction effect $(p=0.002)$, large effect size (Table A.3). Post hoc analysis was performed to find differences between groups, but differences did not exist among two training groups $(p>0.05)$. Additionally, the UPRT group was the only group indicated significant difference with control group $(p=0.04)$ as well as significant changes in preand post-measures $(p<0.001)$, large effect size.

As depicted in Table A.4, there were no statistically significant differences among experimental groups and also between them with control group considering both socio-psychological factors, namely GHQ 
and PSCQ. It should be mentioned that there did not exist significant differences in pre- and postmeasures of experimental groups $(p>0.05)$.

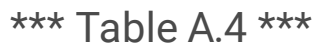

\section{Discussion}

Physical fitness seems to play a relevant role in general health [1], and physical fitness in childhood and adolescence profoundly impact future, adult health. In this vein, RT has emerged as powerful modulator of physical fitness [3]. However, it has not been properly studied whether different programmed approaches to RT with adolescents produce different physical and psychosocial outcomes. The goal of the current study was therefore to compare the effects of 8 weeks' resistance training with linear and undulating programming on physical fitness and mental health of adolescent boys.

Based on the findings, both experimental groups produced significantly greater improvements than the control group in terms of maximal muscle strength. The change percent of upper- and lower extremities strength for LPRT and UPRT groups was $+40.19,+42.16$ and $+102.89,+126.04$, respectively. There were no significant differences between the two programs. These findings are consistent with evidence provided by Niazi, Parnow [30].

Similarly, both experimental groups generated significant improvements in muscle endurance in comparison with controls, but without statistically significant differences between LPRT and UPRT. The change percent for upper- and lower- extremities in LPRT and UPRT groups was $+87.81,+82.08$ and + $86.90,+379.29$, respectively. The findings are in line with previous investigations [30,31]. In the study performed by Niazi, Parnow [30], reported that both upper- and lower- extremities endurance increased following two LPRT and UPRT trainings in untrained female adolescents. Another research showed that two programmed modes of resistance training with equated intensity and volume could significantly change muscle endurance in adolescent athletes [31].

As for balance, there were no significant differences among the three groups considering right leg, although there were meaningful differences were present after evaluating for left leg balance. Again, it was shown that the two programs similarly improved dynamic balance in male adolescents. Previous studies have shown that combined training modalities are superior to balance-specific and plyometric training in terms of balance gains, while all three are superior to RT [32,33].

Explosive power improved in both experimental groups, being statistically significant in comparison with controls, but not between themselves. Our findings are in inconsistent with the results of Niazi, Parnow [30], that have revealed that an 8-week LPRT and UPRT did not change the explosive-power in untrained female adolescents.

None of the programs changed speed levels significantly, in alignment with the research by de Villarreal, Requena [34], and in contradiction with other research [35,36] that has showed the improvement of speed 
by various interventions including plyometric, resistance training and combined intervention trainings. Perhaps the differences may rely in type, intensity and duration of training protocol, as well as in age and baseline level of physical fitness.

Base on the FFMI findings, there were no statistically significant differences between training groups. Consistent with our results, Rhea, Ball [37] compared 12-week resistance training with LPRT and DUP in changing body composition, and reported no significant differences in both groups. In this regard, another research group also did not find any significant differences between linear and non-linear groups after 12-week training [22].

As for the psychosocial factors, there were no significant differences within and between groups in terms of general health and physical self-concept factors. Therefore, both training programs were ineffective in promoting improvements in the analyzed psychosocial factors. Perhaps 8 weeks were not enough for producing significant changes in such complex constructs. Although the change in physical fitness is considered to be a factor capable of modulating self-esteem, initial levels of physical fitness and selfesteem can influence the magnitude of gains [38].

\section{Conclusions}

Resistance training programs were effective in improving several physical fitness outcomes in comparison with controls. However, they were not effective in improving psychosocial markers, which may perhaps be due to the short duration of the protocol (8 weeks). Furthermore, the two programs had no statistically significant differences, suggesting that as long as loads are individually adjusted and progressed, the specific programming adopted may not be relevant for beginner practitioners.

\section{Declarations}

Authors: All authors read and approved the final version of the manuscript.

Financial support: There is no funding to report.

Conflicts of interest: The authors have no conflicts of interest to report.

\section{Authors' contribution}

Abdolhossein Parnow, Masoud Sadeghi, Omid Razi: Research concept and study design, literature review, data collection, data analysis and interpretation, statistical analysis, writing of the manuscript.

Alexandre Martins, José Afonso: Literature review, data analysis and interpretation, reviewing and editing drafts of the manuscript.

\section{ACKNOWLEDGMENTS}


The authors would like to acknowledge the youth athletes involved in this investigation.

\section{References}

1. Ortega FB, Ruiz JR, Castillo MJ, Sjöström M. Physical fitness in childhood and adolescence: a powerful marker of health. International Journal of Obesity. 2008;32(1):1-11.

2. Anderson E, Durstine JL. Physical activity, exercise, and chronic diseases: A brief review. Sports Medicine and Health Science. 2019;1(1):3-10.

3. Dumith SC, Gigante DP, Domingues MR, Kohl III HW. Physical activity change during adolescence: a systematic review and a pooled analysis. International journal of epidemiology. 2011;40(3):685-98.

4. Christou M, Smilios I, Sotiropoulos K, Volaklis K, Pilianidis T, Tokmakidis SP. Effects of resistance training on the physical capacities of adolescent soccer players. The Journal of Strength \& Conditioning Research. 2006;20(4):783-91.

5. Hart PD, Buck DJ. The effect of resistance training on health-related quality of life in older adults: Systematic review and meta-analysis. Health promotion perspectives. 2019;9(1):1.

6. Faigenbaum AD, Kraemer WJ, Blimkie CJ, Jeffreys I, Micheli LJ, Nitka M, et al. Youth resistance training: updated position statement paper from the national strength and conditioning association. The Journal of Strength \& Conditioning Research. 2009;23:S60-S79.

7. Pediatrics AAo. Weight training and weight lifting: information for the pediatrician. The Physician and Sportsmedicine. 1983;11(3):157-61.

8. Ryan JR, Salciccioli GG. Fractures of the distal radial epiphysis in adolescent weight lifters. The American journal of sports medicine. 1976;4(1):26-42.

9. Vrijens J. Muscle strength development in the pre-and post-pubescent age. Pediatric Work Physiology. 11: Karger Publishers; 1978. p. 152-8.

10. Kekäläinen T, Kokko K, Sipilä S, Walker S. Effects of a 9-month resistance training intervention on quality of life, sense of coherence, and depressive symptoms in older adults: randomized controlled trial. Quality of life research. 2018;27(2):455-65.

11. Putiri AL, Gillham S, Ryan Bradley N. Psychological effects of Yi Ren Medical Qigong and progressive resistance training in adults with type 2 diabetes mellitus: a randomized controlled pilot study. Alternative therapies in health and medicine. 2012;18(1):30.

12. Sparrow D, Gottlieb DJ, DeMolles D, Fielding RA. Increases in muscle strength and balance using a resistance training program administered via a telecommunications system in older adults. Journals of Gerontology Series A: Biomedical Sciences and Medical Sciences. 2011;66(11):1251-7.

13. Organization WH. Global recommendations on physical activity for health: World Health Organization; 2010.

14. Peitz $M$, Behringer $M$, Granacher $U$. A systematic review on the effects of resistance and plyometric training on physical fitness in youth-What do comparative studies tell us? PloS One. 2018;13(10).

15. Bompa TO, Buzzichelli C. Periodization-: theory and methodology of training: Human kinetics; 2018. 
16. Rhea MR, Alderman BL. A meta-analysis of periodized versus nonperiodized strength and power training programs. Research quarterly for exercise and sport. 2004;75(4):413-22.

17. Fleck S. Non-linear periodization for general fitness \& athletes. Journal of human kinetics. 2011;29(Special-Issue):41-5.

18. Moraes E, Fleck SJ, Dias MR, Simão R. Effects on strength, power, and flexibility in adolescents of nonperiodized vs. daily nonlinear periodized weight training. The Journal of Strength \& Conditioning Research. 2013;27(12):3310-21.

19. Harries SK, Lubans DR, Callister R. Systematic review and meta-analysis of linear and undulating periodized resistance training programs on muscular strength. The Journal of Strength \& Conditioning Research. 2015;29(4):1113-25.

20. Schumann M, Notbohm H, Bäcker S, Klocke J, Fuhrmann S, Clephas C. Strength-Training Periodization: No Effect on Swimming Performance in Well-Trained Adolescent Swimmers. International Journal of Sports Physiology and Performance. 2020;1(aop):1-9.

21. Grgic J, Lazinica B, Mikulic P, Schoenfeld BJ. Should resistance training programs aimed at muscular hypertrophy be periodized? A systematic review of periodized versus non-periodized approaches. Science \& Sports. 2018;33(3):e97-e104.

22. Prestes J, Frollini AB, de Lima C, Donatto FF, Foschini D, de Cássia Marqueti R, et al. Comparison between linear and daily undulating periodized resistance training to increase strength. The Journal of Strength \& Conditioning Research. 2009;23(9):2437-42.

23. Brzycki M. Strength testing-predicting a one-rep max from reps-to-fatigue. Journal of Physical Education, Recreation \& Dance. 1993;64(1):88-90.

24. Coburn JW, Malek MH. NSCA's Essentials of Personal Training 2nd Edition: Human Kinetics; 2012.

25. Mackenzie B. Performance evaluation tests. London: Electric World plc. 2005;24(25):57-158.

26. Shaffer SW, Teyhen DS, Lorenson CL, Warren RL, Koreerat CM, Straseske CA, et al. Y-balance test: a reliability study involving multiple raters. Military medicine. 2013;178(11):1264-70.

27. Gibson AL, Wagner D, Heyward V. Advanced Fitness Assessment and Exercise Prescription (8th Ed.): Human kinetics; 2018.

28. Eston R, Reilly T. Kinanthropometry and exercise physiology laboratory manual: Tests, procedures and data. Volume two: Physiology (3rd Ed.): Routledge; 2013.

29. Beam JR, Szymanski DJ. Validity of 2 skinfold calipers in estimating percent body fat of collegeaged men and women. The Journal of Strength \& Conditioning Research. 2010;24(12):3448-56.

30. Niazi N, Parnow A, Eslami R. Effects of Programs with linear and nonlinear periodization on muscular strength and endurance in untrained youth girls. 2016.

31. Ramalingam S, Yee K. Comparison of linear and daily undulating periodization with equated volume and intensity for muscular endurance in adolescent athletes. Asian Journal of Exercise \& Sports Science. 2013;10(2):36-48. 
32. Mohammadi V, Alizadeh MH, Gaieni A. The effects of strength, balance and combined (strength and balance) exercise programs on the dynamic balance of young male athletes. Journal of Research in Rehabilitation Sciences. 2013;9(1):113-23.

33. Motalebi SA, Cheong LS, Iranagh JA, Mohammadi F. Effect of low-cost resistance training on lowerlimb strength and balance in institutionalized seniors. Experimental aging research. 2018;44(1):4861.

34. de Villarreal ES, Requena B, Izquierdo M, Gonzalez-Badillo JJ. Enhancing sprint and strength performance: combined versus maximal power, traditional heavy-resistance and plyometric training. Journal of science and medicine in sport. 2013;16(2):146-50.

35. Gnaneshwar M, Gopinath R. Effect of polymeric training isotonic training and combination of plyometric and isotonic training on leg strength and muscular endurance. Int Educ EJ. 2014;3(1):199-202.

36. Argus CK, Gill ND, Keogh JW, McGuigan MR, Hopkins WG. Effects of two contrast training programs on jump performance in rugby union players during a competition phase. International journal of sports physiology and performance. 2012;7(1):68-75.

37. Rhea MR, Ball SD, Phillips WT, Burkett LN. A comparison of linear and daily undulating periodized programs with equated volume and intensity for strength. The Journal of strength \& conditioning research. 2002;16(2):250-5.

38. Spence JC, McGannon KR, Poon P. The Effect of Exercise on Global Self-Esteem: A Quantitative Review. 2005;27(3):311.

\section{Supplementary Files}

This is a list of supplementary files associated with this preprint. Click to download.

- Appendices.docx 